\title{
The effects of levosimendan on brain metabolism during initial recovery from global transient ischaemia/hypoxia
}

Anna B Roehl ${ }^{1 *+}$, Norbert Zoremba ${ }^{1+}$, Markus Kipp $^{2}$, Johannes Schiefer ${ }^{3}$, Andreas Goetzenich ${ }^{1}$, Christian Bleilevens ${ }^{1}$, Nikolaus Kuehn-Velten ${ }^{4}$, Rene Tolba ${ }^{5}$, Rolf Rossaint ${ }^{1}$ and Marc Hein ${ }^{1}$

\begin{abstract}
Backround: Neuroprotective strategies after cardiopulmonary resuscitation are currently the focus of experimental and clinical research. Levosimendan has been proposed as a promising drug candidate because of its cardioprotective properties, improved haemodynamic effects in vivo and reduced traumatic brain injury in vitro. The effects of levosimendan on brain metabolism during and after ischaemia/hypoxia are unknown.

Methods: Transient cerebral ischaemia/hypoxia was induced in 30 male Wistar rats by bilateral common carotid artery clamping for 15 min and concomitant ventilation with $6 \% \mathrm{O}_{2}$ during general anaesthesia with urethane. After 10 min of global ischaemia/hypoxia, the rats were treated with an i.v. bolus of $24 \mathrm{\mu g} \mathrm{kg}^{-1}$ levosimendan followed by a continuous infusion of $0.2 \mathrm{\mu g} \mathrm{kg}^{-1} \mathrm{~min}^{-1}$. The changes in the energy-related metabolites lactate, the lactate/ pyruvate ratio, glucose and glutamate were monitored by microdialysis. In addition, the effects on global haemodynamics, cerebral perfusion and autoregulation, oedema and expression of proinflammatory genes in the neocortex were assessed.

Results: Levosimendan reduced blood pressure during initial reperfusion ( $72 \pm 14$ vs. $109 \pm 2 \mathrm{mmHg}, \mathrm{p}=0.03$ ) and delayed flow maximum by 5 minutes $(p=0.002)$. Whereas no effects on time course of lactate, glucose, pyruvate and glutamate concentrations in the dialysate could be observed, the lactate/pyruvate ratio during initial reperfusion ( $144 \pm 31$ vs. $77 \pm 8, p=0.017)$ and the glutamate release during 90 minutes of reperfusion ( $75 \pm 19$ vs. $24 \pm 28 \mathrm{\mu mol} \cdot \mathrm{L}^{-1}$ ) were higher in the levosimendan group. The increased expression of IL-6, IL-1B TNFa and ICAM-1, extend of cerebral edema and cerebral autoregulation was not influenced by levosimendan.

Conclusion: Although levosimendan has neuroprotective actions in vitro and on the spinal cord in vivo and has been shown to cross the blood-brain barrier, the present results showed that levosimendan did not reduce the initial neuronal injury after transient ischaemia/hypoxia.
\end{abstract}

Keywords: Levosimendan, Cerebral ischaemia, Hypoxia, Microdialysis

\section{Background}

Patients under cardiac arrest undergo acute global ischaemia and acute reperfusion injury due to the return of spontaneous circulation. Although this reperfusion injury affects all organs, the heart and brain are particularly vulnerable. Neurological and cardiac complications

\footnotetext{
*Correspondence: aroeh|@ukaachen.de

${ }^{\dagger}$ Equal contributors

'Department of Anaesthesiology, RWTH Aachen University Hospital, Pauwelstrasse 30, Aachen D-52074, Germany

Full list of author information is available at the end of the article
}

following cardiopulmonary resuscitation (CPR) are closely associated and might aggravate cellular damage. Cerebral ischaemia activates cellular processes, including apoptosis, inflammation, inhibition of protein synthesis and increased oxidative stress, that persist despite the restoration of substrate delivery [1]. This initial neuronal injury involves disruptions in brain metabolism and the release of neurotransmitters, which activate neurotoxic cascades that can be monitored by microdialysis [2-4].

Studies have previously demonstrated that ischaemic postconditioning dramatically attenuates irreversible

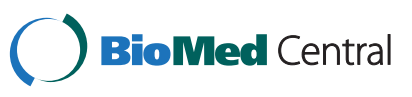


myocardial injury [5]. Furthermore, a reduction of the cerebral ischaemia-reperfusion injury due to ischaemic postconditioning has been previously described [6]. Interestingly, studies have estimated that every minute of lost cerebral perfusion in the human brain results in a loss of neurons that is equivalent to the loss of neurons after 3.6 years of the normal human ageing process [7]. Currently, the only valid form of therapy to improve neurological outcome is to perform therapeutic hypothermia 12 to $24 \mathrm{~h}$ following resuscitation [8]. Concomitant with the symptomatic therapy used to achieve return of spontaneous circulation (ROSC), therapeutic options employed to minimise the reperfusion injury have to be considered.

The ideal pharmacological intervention would be initiated during CPR and would exhibit cardiac and neuroprotective properties. Thus, a cardiac postconditioning effect may only be attained prior to the time window because the cardiac postconditioning closes two minutes after the re-establishment of spontaneous circulation [9]. The time frame for cerebral postconditioning has previously been described as a maximum of three minutes following the onset of reperfusion [10], whereas expansion of the penumbra after focal ischaemia may be reduced within the first six hours after reperfusion. Thus, sufficient effect-site concentrations of postconditioning agents should be made available at the start of the reperfusion. Levosimendan is a novel inodilator that enhances myocardial performance without resulting in substantial changes in myocardial oxygen consumption [11]. Levosimendan reduces myocardial injury if it is applied during ischaemia and early reperfusion [12]. Evidence of levosimendan's neuroprotective properties include reduced cell death, inflammatory response and lipid peroxidation in the spinal cord and improved function after transient ischaemia [13,14]. Currently, the demonstration of a reduction of primary and secondary injury after brain trauma has been limited to in vitro models [15]. The protective effects of levosimendan are mediated in the heart by activation of the PI3K pathway, the inducible nitric oxide synthase and mitochondrial ATP-dependent potassium channels $\left(\mathrm{mK}_{\mathrm{ATP}}\right)$. This also results in a clearly vasodilation [16-18]. The important role of $\mathrm{mK}_{\mathrm{ATP}}$ channels in cerebral ischaemia-reperfusion injury and positive action of other activators (diazoxide) on neuronal injury, spark hopes of neuroprotective effects of levosimendan $[19,20]$.

Based on the promising results of levosimendan, the aim of the present study was to test the hypothesis that levosimendan reduces initial ischaemic/hypoxic neuronal injury in the neocortex by postconditioning. We employed a model of bilateral carotid occlusion with an additional reduction of inspired oxygen concentration. Changes in the metabolite and substrate levels, haemodynamics, regional perfusion, blood-brain barrier dysfunction and local inflammatory response were measured by microdialysis, blood pressure measurements, laser Doppler flow, and claudin 5/tight junction protein and interleukin expression, respectively.

\section{Methods}

\section{Instrumentation}

All of the experiments were performed in accordance with the German legislation governing animal studies and followed the Guide for the Care and Use of Laboratory Animals [21]. Official permission for these studies was granted from the governmental animal care and use office (Landesamt für Natur-, Umwelt- und Verbraucherschutz Nordrhein-Westfalen, Recklinghausen, Germany, Protocol No. 8.87-50.10.55.09.064).

A previously established protocol was modified to investigate the effects of levosimendan on ischaemic brain injury [22]. Thirty male Wistar rats (Charles River, Sulzfeld, Germany, 300-350 g) were anaesthetised by an intraperitoneal injection of $1.5 \mathrm{~g} / \mathrm{kg}$ urethane (SigmaAldrich Chemie GmbH, Steinheim, Germany). After a tracheotomy, the animals were ventilated with $30 \%$ oxygen under the pressure-controlled mode $\left(\mathrm{P}_{\max } 13 \mathrm{mmHg}\right.$, PEEP $5 \mathrm{~mm} \mathrm{Hg}$ ) using a commercially available respirator (Evita 2, Draeger, Luebeck, Germany) with a respiratory frequency between 40 and $55 \mathrm{~min}^{-1}$. The right femoral vein was catheterised with a 22 G cannula (Leader Flex, Vygon, Germany) using Seldinger's technique for continuous drug administration and blood withdrawal. The mean arterial pressure (MAP) was measured with a 1.4 F pressure catheter (SPR-671, Millar Instruments, Houston, Texas, USA) that was placed through the right femoral artery. The heart rate (HR) was calculated from the ECG signal. All of the data were recorded using an acquisition and analysis system (Power Lab 8/30, LabChart 6 Pro v 6.11; ADInstruments, Colorado Springs, USA). A continuous infusion of $4 \mathrm{ml} \mathrm{kg}^{-1} \mathrm{~h}^{-1}$ Ringers solution was administered to compensate for the perioperative fluid loss. To maintain body temperature, the animals were placed on a backcoupled heating pad (MLT1403 and TCAT-2 Controller, ML 295/R, Physitemp Instruments, USA). Both of the common carotid arteries were identified in the supine position and isolated from the attached vagal nerve. To induce carotid clamping, a 2-0 silk suture (Fine Science Tools, Heidelberg, Germany) was placed around each common carotid artery.

In the prone position, the heads of the rats were fixed in a stereotactic apparatus (Figure 1). A laser Doppler probe (moor VMS-LDF1, Moor Instruments Inc., Devon, Great Britain) was positioned $5.5 \mathrm{~mm}$ lateralright and $1 \mathrm{~mm}$ caudal from the bregma. The cranium was thinned using a small hand drill (Dremel 300 Series, 


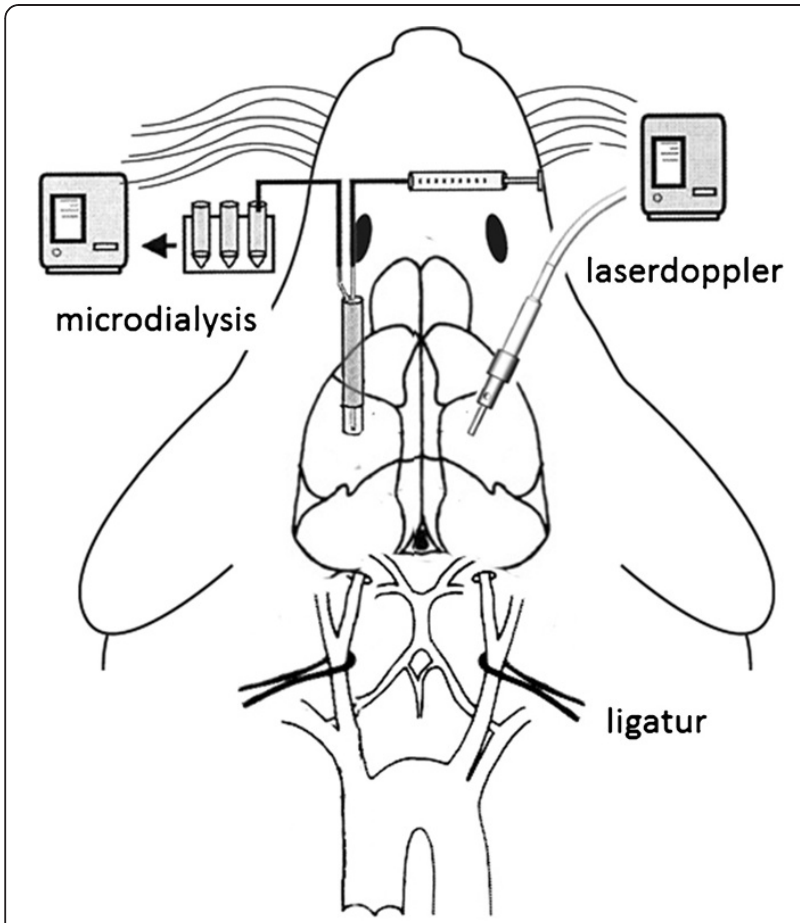

Figure 1 Schematic of the experimental setting.

Dremel, Leinfeld-Echterdingen, Germany), and the laser Doppler probe was positioned in a vertical position and fixed with a commercially available instant adhesive (UHU, Bühl, Germany) and activator spray (JAMARA Modeltechnik, Aichstetten, Germany). The recorded perfusion units (PFU) were normalised (100\%) to values at baseline that were recorded $10 \mathrm{~min}$ prior to the induction of ischaemia/hypoxia. The impairment of cerebral autoregulation was characterised by the autoregulatory index (ARI), which was calculated from the slope obtained from the linear regression analysis of the relative change of PFU (\%) and MAP (\%) during reperfusion [23]. The cerebral vascular resistance (CVR) was calculated as the ratio of the MAP to the normalised PFU.

The left somatosensory cortex was partially exposed by a burr hole located 2-3 mm caudal from the bregma and 3-4 $\mathrm{mm}$ lateral from the midline (Figure 1). The dura was partially opened with a needle to place a microdialysis probe with a membrane length of $2 \mathrm{~mm}$, an outer diameter of $0.5 \mathrm{~mm}$ and a cut-off at 20000 DA (CMA 12, 2 mm membrane length, CMA Microdialysis, Solna, Sweden). The microdialysis method has been previously described in detail by Ungerstedt et al. [24]. The microdialysis catheter was continuously perfused with a dialysate containing $147 \mathrm{mmol} / \mathrm{l} \mathrm{NaCl}, 2.7 \mathrm{mmol} / \mathrm{l} \mathrm{KCl}$, $1.2 \mathrm{mmol} / \mathrm{l} \mathrm{CaCl}_{2}$ and $0.85 \mathrm{mmol} / \mathrm{l} \mathrm{MgCl}_{2}$ (Perfusion fluid CNS, CMA Microdialysis) at a flowrate of $2 \mu \mathrm{l}^{*} \mathrm{~min}^{-1}$ using a precision infusion pump (CMA 102, CMA Microdialysis). The samples were collected in
10 min intervals and frozen at $-20^{\circ} \mathrm{C}$ until the analysis. The thawed and centrifuged dialysate samples were analysed enzymatically with a chemistry analyser (CMA 600 Microdialysis Analyser, CMA Microdialysis, Schweden) for lactate, pyruvate, glucose and glutamate concentrations. Prior to the experiments and at the end of the experiments, the relative recovery rates for each substance were determined with a calibration solution (Calibrator, CMA Microdialysis) and applied to the experimental values. For glutamate, the area under the concentration curve was calculated to quantify the relationship between release and uptake.

\section{Experimental protocol}

Due to the transient increases in metabolite concentrations from placing the microdialysis probe in the brain, an equilibration period of $60 \mathrm{~min}$ was required. Three baseline measurements of haemodynamic data and corresponding cerebral microdialysates were each sampled within a $10 \mathrm{~min}$ interval. To prevent the rats from spontaneous breathing triggered by hypoxia, $2 \mathrm{mg} \mathrm{kg}-1$ of rocuronium (Esmeron, Schering-Plough, Kenilworth, NJ, USA) was injected i.v. The rats were randomly divided into three groups using an envelope system. After $10 \mathrm{~min}$ of ischaemia, the first group received a bolus of $24 \mathrm{\mu g} \mathrm{kg}^{-1}$ levosimendan (Simdax ${ }^{\circledR} 2.5 \mathrm{mg} / \mathrm{ml}$, Orion Pharma, Espoo, Finland), which was administered over a period of $20 \mathrm{~min}$, followed by a continuous infusion of $0.2 \mu \mathrm{g} \mathrm{kg}^{-1} \mathrm{~min}^{-1}$ throughout the experiment. The control group received an equivalent amount of $0.9 \% \mathrm{NaCl}$. The sham rats were not subjected to cerebral ischaemia/hypoxia but did receive an equivalent amount of $\mathrm{NaCl}$ as the control group. To induce ischaemia and hypoxia, both ligatures around the carotid arteries were closed, and the inspired oxygen concentration was reduced to $6 \%$. Although the haemodynamic variables were recorded during ischaemia/ hypoxia within a $5 \mathrm{~min}$ interval, the dialysate was collected over the entire $15 \mathrm{~min}$ of hypoxia/ischaemia. After $15 \mathrm{~min}$, the ligatures were released, and the inspired oxygen concentration values normalised (30\%). During the following $90 \mathrm{~min}$ of reperfusion, the data were recorded every $10 \mathrm{~min}$, and the dialysate was also collected during these intervals.

At the end of the measurement period, the abdomen and thoracic cavity were opened while the rat was in the supine position. The left ventricle of the beating heart was cannulated (22 G, Microlance, Becton Dickinson, Espana), and $4 \mathrm{ml}$ of blood was collected for further analysis. The animals were transcardially perfused with $50 \mathrm{ml}$ of cold $\left(4^{\circ} \mathrm{C}\right)$ Ringers solution. The brain was excised and cut into seven $2 \mathrm{~mm}$ cross-sectional slices. Two slices were used to calculate the percentage of water content, and 2 slices (the fourth and fifth) were 
immediately snap-frozen in liquid nitrogen and stored at $-80^{\circ} \mathrm{C}$ for real-time PCR examination. The rest of the slices were used for further tissue analysis of the levosimendan concentration (Figure 2).

\section{Levosimendan brain tissue concentration}

The brain tissue samples were homogenised in $1 \mathrm{ml}$ of TRIS buffer (Tris-Base $20 \mathrm{mmol} / \mathrm{l} \mathrm{mM}, \mathrm{NaCl} 150 \mathrm{mM}$, EDTA $1 \mathrm{mM})$ per gram of wet weight. The homogenates $(500 \mu \mathrm{l}$ per $50 \mathrm{mg}$ of tissue) were extracted at a neutral $\mathrm{pH}$ with ethyl acetate. After evaporation and resuspension in the mobile phase (acetonitrile/1\% acetic acid), separation of levosimendan was achieved using a reversed-phase $\mathrm{C} 18$ column (the retention time was $1.4 \mathrm{~min}$ ) with zonisamide as the internal standard. Specific ions were detected with an API4000 tandem MS/MS in the negative multiple reaction mode (Sciex/ Applied Biosystems, Foster City, CA, USA).

\section{S100ß protein analysis}

S100ß serum levels at the end of the reperfusion were analysed by an enzyme-linked immunosorbent assay (ELISA) using a commercially available kit. The assays were performed according to the manufacturer's instructions (YK150, SCETI, Tokyo, Japan).

\section{Messenger RNA quantification by real-time RT-PCR}

Gene expression levels of the pro-inflammatory cytokines tumour necrosis factor $\alpha$ (TNF $\alpha$ ), interleukin 6 (IL-6), interleukin $1 \beta(I L-1 \beta)$ and intercellular adhesion molecule 1 (ICAM-1) in the cortex close to the laser Doppler position were determined by quantitative realtime polymerase chain reaction (qRT-PCR). Disruption of the blood-brain barrier was assessed by the gene expression of claudin 5 (Cldn-5) and tight junction protein 1 (Tjp-1). Total RNA was extracted using a commercially available RNA/Protein extraction kit (NucleoSpin ${ }^{\circledR}$ RNA/Protein, Machery-Nagel, Düren, Germany) and reverse-transcribed into cDNA using a high-capacity reverse transcription kit (Applied Biosystems $^{\circledR}$, Carlsbad, CA, USA). The PCR reaction was performed using $50 \mathrm{ng}$ of cDNA (TaqMan ${ }^{\circledR}$ universal PCR mix, Applied Biosystems ${ }^{\circledR}$ ) and specific TaqMan ${ }^{\circledR}$ probes for IL-1 $\beta$ (Rn00580432_m1), IL-6 (Rn01410330_m1), TNF- $\alpha$ (Rn00562055_m1), ICAM-1 (Rn00564227_m1), Cldn-5 (Rn01753146_s1), Tjp-1 (Rn02116071_s1) and the housekeeping gene hypoxanthin-guanin-phosphoribosyltransferase (HPRT, Rn01527840_m1) on a StepOnePlus ${ }^{\circledR}$ Cycler (Applied Biosystems ${ }^{\circledR}$ ). The relative quantity (RQ) values were calculated according to the $\Delta \Delta \mathrm{Ct}$ method, which reflects the differences in the threshold for each target gene relative to HPRT and the shamoperated rat.

\section{Statistical analysis}

We used a multivariate analysis for repeated measurements and a univariate analysis using Scheffe's or Kruskal-Wallis post hoc test for pairwise comparisons between the groups (depending on the results obtained from Levene's test for the equality of variances). A onesample $t$ test was used to compare the RQ values with the sham-operated rats $(R Q=1)$. The effects of ischaemia/hypoxia and levosimendan on cerebral autoregulation were determined using multiple linear regression analysis (SPSS 19; IBM Corporation, Somers, NY, USA). The RQ values were plotted as box and whisker graphs displaying the 5th and 95th percentiles, whereas all of the other results were presented as the mean and SEM (Prism 5.01, GraphPad Software, San Diego, CA). A p-value of $<0.05$ was considered to be statistically significant.

\section{Results}

Six of the 30 rats died during the experimental procedure because of several factors, including procedureinduced injury of the carotid artery, cardiac failure, ventricular fibrillation and brain death. Nine rats were treated with levosimendan, 9 rats received $\mathrm{NaCl}$ (control group) and sham-operations were performed on 6 rats. Levosimendan crossed the blood-brain barrier and reached a tissue concentration of $0.17 \pm 0.13 \mathrm{ng} \mathrm{g}^{-1}$.

\section{The effects of ischaemia/hypoxia}

During ischaemia/hypoxia, the HR decreased from $407 \pm 1$ to $325 \pm 10 \mathrm{~min}^{-1}$ and MAP from $84 \pm 3$ to

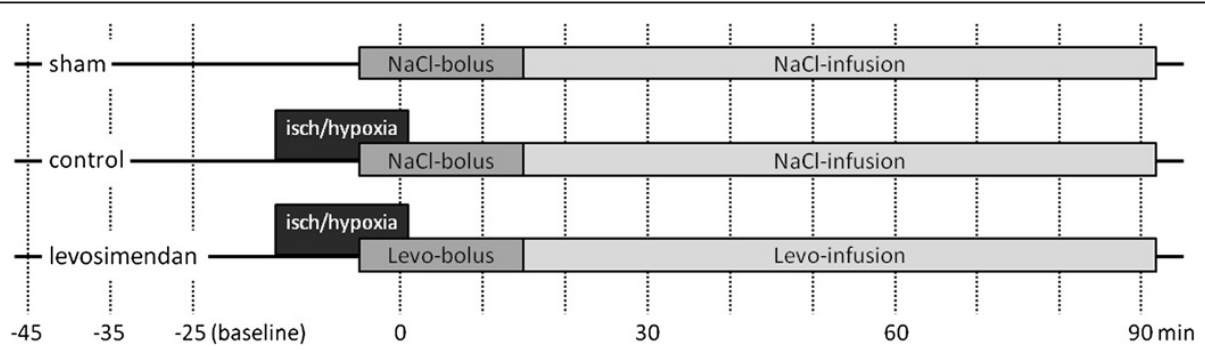

Figure 2 A study design diagram: the treatment of the groups related to the procedures. The vertical lines mark the measurement time points. 
$32 \pm 2 \mathrm{mmHg}$ with no differences between the levosimendan and the control group. The LDF signal had dropped to $6-8 \%$ of the baseline values in both of the groups. No effect of the levosimendan bolus during this period was evident (Figure 3). Both, the levosimendan as well as the control group showed a relevant increase in lactate $\left(+1282 \pm 86 \mu \mathrm{mol} \mathrm{L^{-1 } )}\right.$ and glutamate $\left.(+37 \pm 1 \mu \mathrm{mol} \mathrm{L})^{-1}\right)$ levels, whereas the levels of pyruvate $\left(-16 \pm 2 \mu \mathrm{mol} \mathrm{L}^{-1}\right)$ and glucose $\left(-1391 \pm 158 \mu \mathrm{mol} \mathrm{L}{ }^{-1}\right)$ were markedly decreased. Thus, the lactate/pyruvate ratio increased by 5 times during ischaemia/hypoxia (Figure 4). No differences were found between the groups.

\section{The effects of reperfusion}

During early reperfusion, an overshoot in the HR, MAP and LDF may be detected with peak values after 10-25 min (Figure 3). The levosimendan group showed a significant delayed (5 min) and smaller increase in the MAP compared with the control group (72 \pm 14 vs. $109 \pm 2 \mathrm{mmHg}, \mathrm{p}=0.03$ ). In addition, the LDF increase to the maximum values was delayed by $5 \mathrm{~min}$ in the levosimendan group $(\mathrm{p}=0.002)$ but reached levels that were comparable with the control group. Moreover, the mean arterial pressure of the levosimendan-treated rats remained $12 \pm 2 \mathrm{mmHg} \quad(\mathrm{p}=0.03)$ lower throughout the reperfusion period, whereas no differences in the LDF amplitudes could be measured between the groups. Ischaemia/hypoxia in both groups resulted in an impairment in the cerebral autoregulation, which was demonstrated by a higher ARI in the control (1.4) and levosimendan (1.72) group compared with the sham group $(0.38, \mathrm{p}<0.001)$. There were no differences between the treatment groups (Figure 5). Although the elevation of the regression line between the LDF and the MAP was significantly different between the groups, no effect of levosimendan on the CVR was observed (Figure 4D). The HR normalised after $90 \mathrm{~min}$ and there were no differences between the groups.

Reperfusion resulted in a further increase in the concentration of lactate and pyruvate in the dialysate within the first $20 \mathrm{~min}$, but this increase was followed by a continuous decrease (Figure $4 \mathrm{~A}$ and $\mathrm{C}$ ). In addition, the lactate/pyruvate ratio decreased less during early reperfusion following levosimendan treatment compared with the control group $(144 \pm 31$ vs. $77 \pm 8, p=0.017$, Figure 4E). Moreover, the glutamate returned to baseline values within $20 \mathrm{~min}$ of reperfusion, whereas glucose concentrations returned to baseline values within $50 \mathrm{~min}$ of reperfusion. Similar to the pyruvate levels, the glucose levels transiently increased within the first 20 min. Furthermore, the levosimendan-treated animals displayed more glutamate release $\left(75 \pm 19 \mu \mathrm{mol} \cdot \mathrm{L}^{-1} 1\right)$ compared with the control $\left(24 \pm 28 \mu \mathrm{mol} \cdot \mathrm{L}^{-1}, \mathrm{p}=0.29\right)$ and sham $\left(-19 \pm 11 \mu \mathrm{mol} \cdot \mathrm{L}^{-1}, \mathrm{p}=0.02\right.$, Figure 4D).

Despite the higher levels of $s 100 ß$ after ischaemia/ hypoxia, no significant differences between the groups
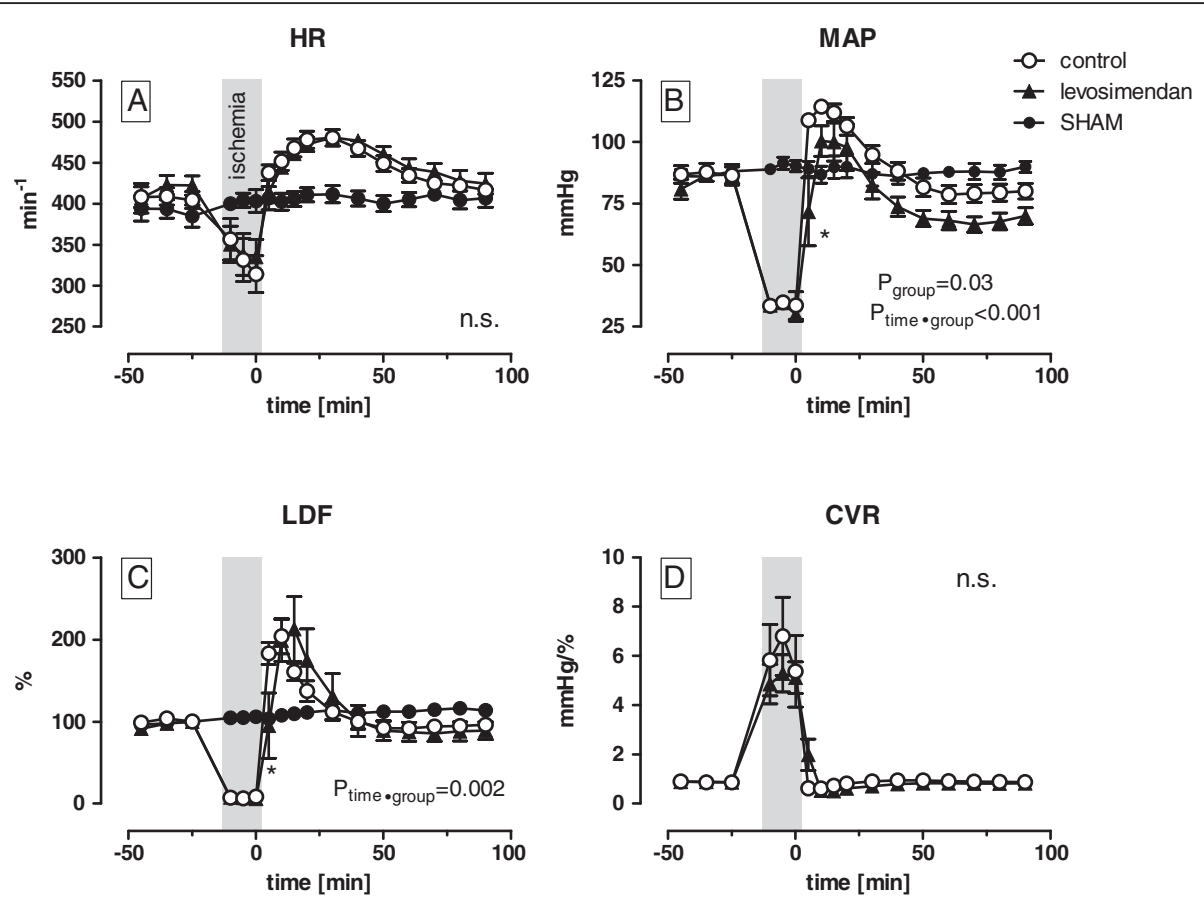

Figuer 3 The effects of levosimendan on the haemodynamics and cerebral perfusion during 15 min of bilateral cerebral ischaemia/hypoxia and $90 \mathrm{~min}$ of reperfusion (the p-values indicate differences between the control and levosimendan treatment group as assessed. 


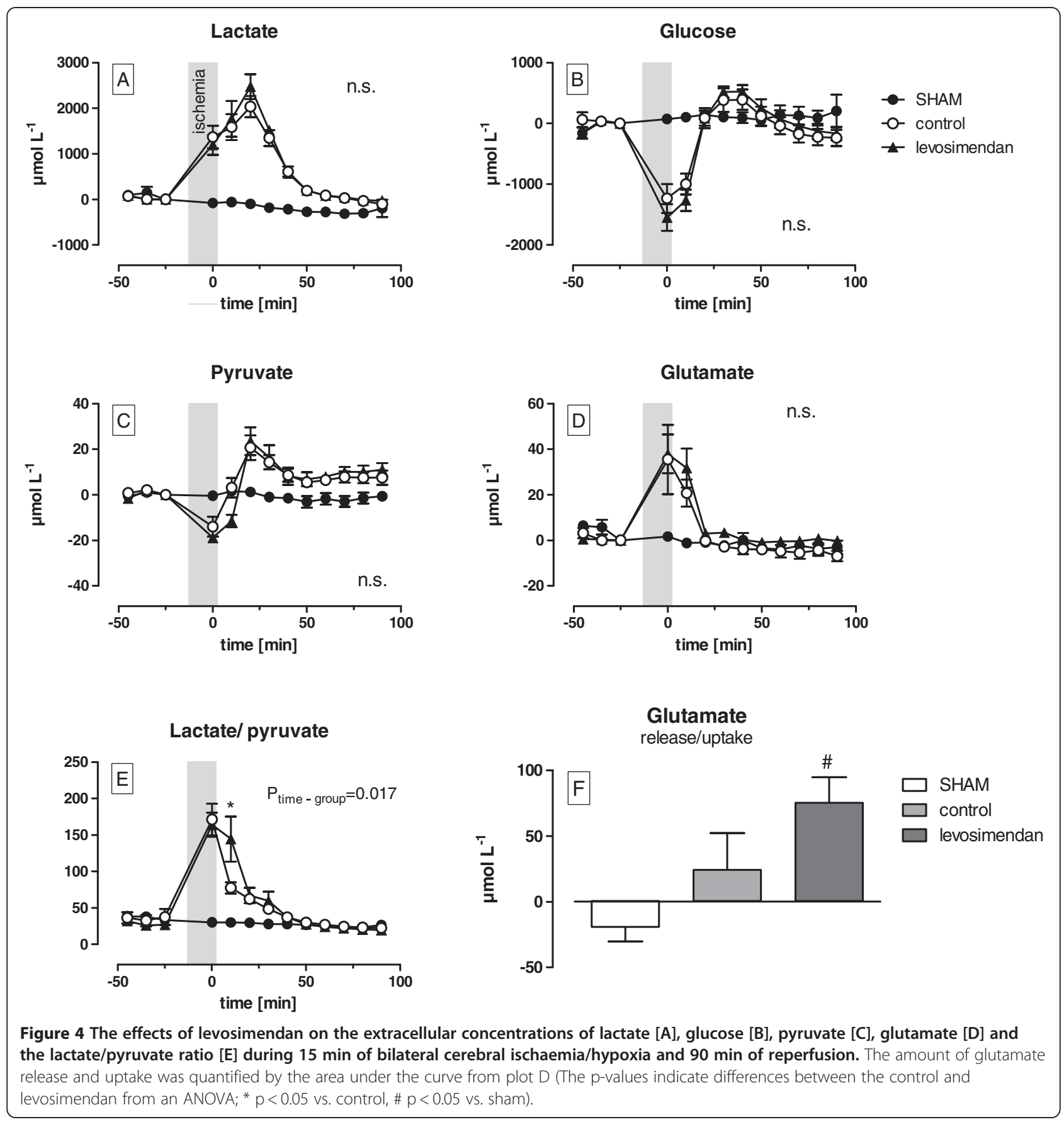

could be detected after 90 min of reperfusion (Figure 6A). Although the water content increased significantly from $68 \pm 7 \%$ in the sham group to $76 \pm 3 \%$ after ischaemia/hypoxia $(\mathrm{p}=0.007)$, there were no differences between the levosimendan-treated group and the control group (Figure 6B). Interestingly, cerebral oedema may be a consequence of reduced expression of Tjp-1 $(\mathrm{RQ}=0.84 \pm 0.03, \mathrm{p}=0.01$, Figure $7 \mathrm{~A})$. In addition, we observed reduced expression of $C l d n-5 R Q$, but the results were not significant $(R Q=0.88 \pm 0.07, p=0.09$, Figure 7B). Ischaemia/hypoxia also led to an increased inflammatory response in the cortex after $90 \mathrm{~min}$ of reperfusion. Indeed, we observed pronounced upregulation of $T N F \alpha$ and $I L-1 \beta$ by factor $62(\mathrm{p}=0.01)$ respectively $8(\mathrm{p}=0.02)$ and a moderate threefold upregulation of ICAM-1 $(\mathrm{p}=0.03)$ in both of the groups, whereas $I L-6$ levels were only elevated by factor 3.5 in the levosimendan-treated group $(\mathrm{p}=0.02)$. Beside this, 

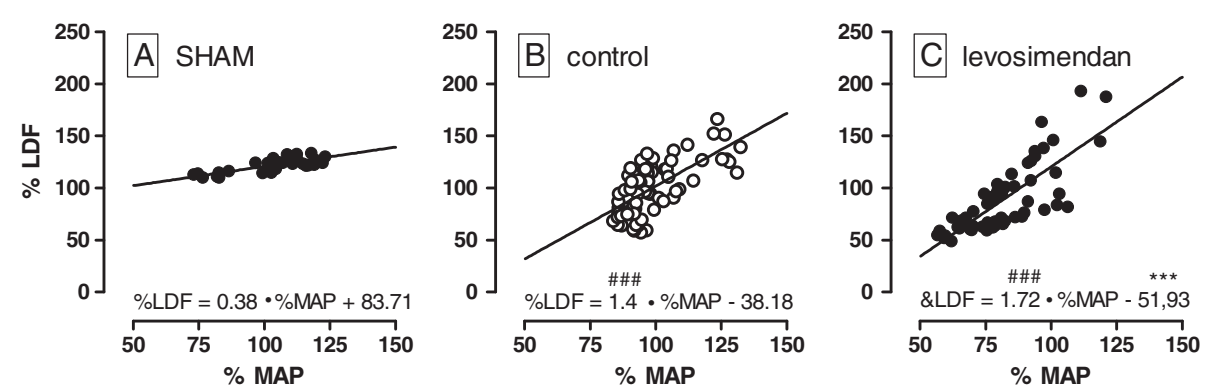

Figure 5 A correlation of the relative change of the laser Doppler flow (\%LDF) and the mean arterial pressure (\%MAP) during reperfusion. The slope of the linear regression reflects the autoregulatory index (\#\#\# $p<0.001$ vs. sham; ${ }^{* *} P<0.001$ vs. control for the intercept). by an ANOVA and post hoc test at various time points, $\left.{ }^{*} \mathrm{p}<0.05\right)$. HR - heart rate, MAP - mean arterial pressure, LDF - laser Doppler flow, CVR - cerebral vascular resistance.

levosimendan did not cause any further significant effects on gene expression compared with the control group (Figure 7).

\section{Discussion}

The aim of the present study was to evaluate the effects of levosimendan on brain metabolism, perfusion and inflammatory response resulting from a defined hypoxia/ ischaemia injury and to characterise the time-course during subsequent re-oxygenation. We found that levosimendan did not reduce the initial ischaemic/hypoxic neuronal injury if it was administered after the insult and during resuscitation.

Ischaemia/hypoxia leads to a primary energy failure that is accompanied by the dysfunction of ATP-dependent ion channels $\left(\mathrm{Na}^{+} / \mathrm{Ca}^{2+}, \mathrm{Ca}^{2+} / \mathrm{H}^{+}\right)$and an increased intracellular $\mathrm{Ca}^{2+}$ concentration $[25,26]$. Subsequent glutamate release activates postsynaptic AMPA and NMDA receptors, which induce intracellular sodium and calcium overflow that may be detected prior to anoxic depolarisation [27]. As a consequence of all this cellular oedema and the activation of different proteases, lipases, endonucleases and the generation of free radicals is induced. Importantly, the restoration of blood flow and oxygenation will restore oxidative metabolism within 30 to $60 \mathrm{~min}$, which suggests a therapeutic window during which the neurotoxic cascade can be inhibited. Most neurons will die as a consequence of secondary energy failure, which occurs 6 to $15 \mathrm{~h}$ after injury [4]. The magnitude of primary cell death is dependent on the severity and duration of ischaemia/hypoxia and could not be observed in the neocortex within the first $13 \mathrm{~min}$ [28]. In the present study, we found that an insignificant increase of s100ß could be observed after 90 min of reperfusion. Thus, the neuroprotective effects of levosimendan observed in the neocortex within the first few hours could only be evaluated based on the monitoring of triggers of cell death (i.e., glutamate release, energy metabolism and inflammation).
Previous studies have demonstrated that it is possible to reduce glutamate release during ischaemia/hypoxia by treatment with tiagabine [29], dantrolene [30], nimodipine [31] or magnesium [32]. In principle, the activation of $\mathrm{mK}_{\text {АTP }}$ channels should result in smaller increases in intracellular $\mathrm{Ca}^{2+}$ levels and glutamate release during ischaemia. Thus, levosimendan may be as effective as diazoxide $[33,34]$. Studies have suggested that $\mathrm{mK}_{\mathrm{ATP}}$ agonists may be beneficial for the treatment of brain disorders that are associated with low ATP levels [35], and levosimendan has been shown to be neuroprotective in the spinal cord when applied prior to [13] or during ischaemia [14]. Preservation of the energy status displayed an important mechanism of protection, but was independent of vasodilatation [36].

The failure of levosimendan to affect neuroprotection the parameters determined during the present study may be related to the low intracerebral concentrations achieved in the current protocol. Although efficient serum concentrations for cardiac effects $(32 \mu \mathrm{g} / \mathrm{L})$ were achieved, the tissue concentration in the brain reached only $12 \%$ of the concentration in the heart. Although this concentration $\left(0.17 \mathrm{ng} \mathrm{g}^{-1}\right)$ was six times higher compared with animals with an intact blood-brain barrier [37], it was not sufficient in the in vitro model to reduce the primary or secondary injury after trauma. In the traumatic brain model injury model, a 100-fold higher concentration was required to achieve significant affects [15]. The low cerebral concentrations could be a consequence of the rapid redistribution of the levosimendan bolus [37] and the delayed disruption of the blood-brain barrier related to the insult [22,38], which appears to be a prerequisite to achieve higher levosimendan levels within the brain.

Other reasons for the ineffectiveness of levosimendan might be related to the unique differences of individual animal models, such as the observation period after the insult, the methods used to describe the neuronal injury and the region of interest. For example, the basal ganglia 


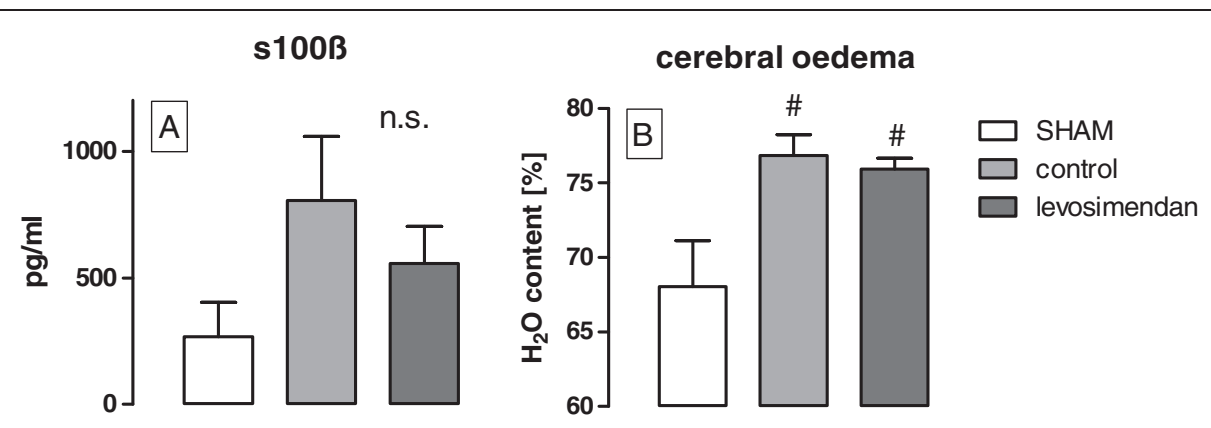

Figure 6 Serum levels of $s 100 ß$ after 90 min of reperfusion (A) and the extent of cerebral oedema characterised by the water content (B) (\# $p<0.05$ vs. sham).

and the hippocampus are more susceptible to ischaemia [28]. The proposed protective effects of levosimendan might become visible after a longer observation period and may not be associated with reduced glutamate release [39]. The magnitude of neuronal injury after $15 \mathrm{~min}$ of ischaemia/hypoxia should be questioned because no lasting effect on metabolism, glutamate release or s100ß increase was observed. Indeed, only the

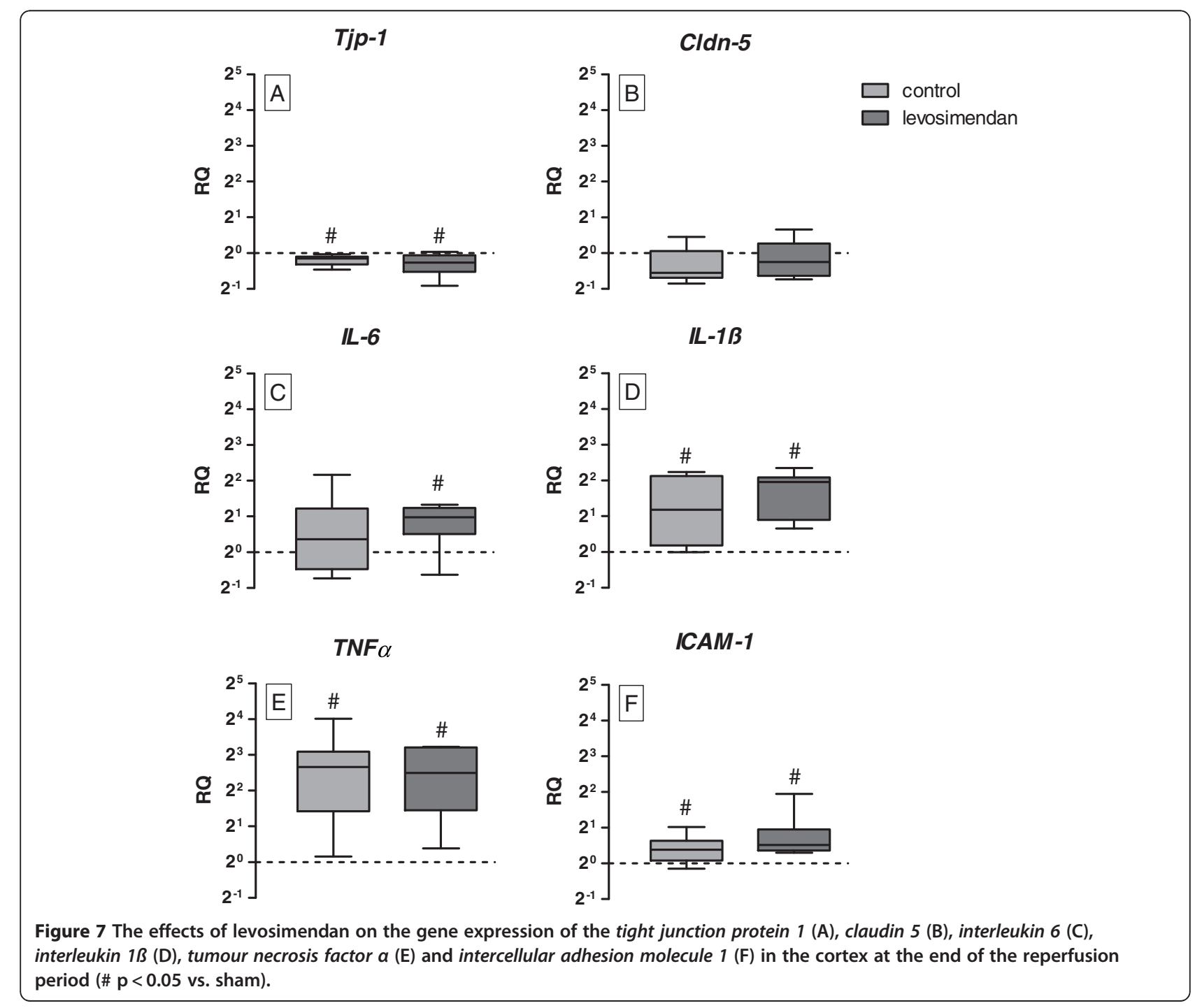


increase in inflammation and cerebral oedema and the disruption of auto regulation and the blood-brain barrier account for cerebral injury. However, if the ischae$\mathrm{mia} /$ hypoxia is prolonged in this model, animals may die from cardiovascular complications or brain death. Thus, modifications to the current protocol for levosimendan administration and a longer observation period are necessary for further validation.

The neuroprotective effects of different drugs might be related to effects on the vasculature. For example, the protective effects of nimodipine and magnesium are associated with decreased cerebral blood flow during reperfusion. Studies have previously shown that controlled reperfusion alone can reduce neuronal injury [40]; thus, the protective actions of drugs may be mediated by a similar mechanism. Indeed, levosimendan affects cerebral perfusion pressure and flow [41]. Although levosimendan delayed reperfusion, we observed an upward shift in the relationship between $\mathrm{CBF}$ and MAP, which might counterbalance levosimendan's direct protective actions. In this context the activation of $\mathrm{mK}_{\mathrm{ATP}}$ channels and increase of NO release in the vessels by levosimendan was effective and led to vasodilation in the brain as described earlier in an animal model of subarachnoid haemorrhage [42]. In addition, the delayed reperfusion during levosimendan treatment may explain the slower normalisation of the lactate/pyruvate ratio. The relevance of this difference is questionable, however, because no differences in the extracellular glucose concentrations were observed.

Other properties associated with cerebral injury were unaffected by levosimendan treatment. The lack of a disturbance of cerebral auto regulation and disruption of the blood-brain barrier resulted in similar cerebral oedema responses between the groups. Similarly, diazoxide also demonstrated protective effects in this context [43], where activation of $K_{\text {ATP }}$ channels reduced the permeability of the $\mathrm{BBB}$ and down regulation of occludin after hypoxia [44]. These differences might be explained by a diverse affinity to receptor subtypes of the substances, which have not been investigated in detail.

Although levosimendan demonstrated anti-inflammatory actions in sepsis, myocardial reperfusion injury and ARDS, no effects on the expression of inflammatory genes in the neocortex were observed. Because inflammation aggravates neuronal injury [45], the protective effects of levosimendan under inflammatory conditions are more unlikely.

\section{Conclusion}

In conclusion, levosimendan did not exhibit neuroprotective actions in the initial phase after experimental ischaemic/hypoxic cerebral injury. We did not find any relevant effects on metabolism, release of glutamate, inflammation, auto regulation or the integrity of the blood-brain barrier. Moreover, no aggravation of brain injury was found.

\section{Competing interests}

The authors declare that they have no competing interest.

\section{Authors' contributions}

$\mathrm{AR}, \mathrm{NZ}$ and $\mathrm{MH}$ conceived of the study, participated in the study's design and coordination, performed the statistical analysis and drafted the manuscript. AR, NZ, CB and MH conducted the experimental laboratory work. RR and RT helped to draft the manuscript. MK, JS and AG participated in the study's design and helped to draft the manuscript. WKV established new laboratory measurements for levosimendan detection and helped to draft the manuscript. All authors read and approved the final manuscript.

\section{Acknowledgements}

This work was supported by the Medical Faculty RWTH Aachen (START grant number 10/2010). We would like to specifically thank Renate Nadenau and Christian Beckers (Department of Anaesthesiology) for their help in our laboratory and Dr. Hartmut Kirchherr (MLHB) for the HPLC-MS/MS analyses.

\section{Author details}

${ }^{1}$ Department of Anaesthesiology, RWTH Aachen University Hospital, Pauwelstrasse 30, Aachen D-52074, Germany. ${ }^{2}$ Department of Neuroanatomy, RWTH Aachen, Wendlingweg 2, Aachen 52072, Germany. ${ }^{3}$ Department of Neurology, RWTH Aachen University Hospital, Pauwelstrasse 30, Aachen 52074, Germany. ${ }^{4}$ MLHB, Medical Laboratory Bremen, Haferwende 12, Bremen 28357, Germany. ${ }^{5}$ Department of Experimental Animal Science, RWTH Aachen University Hospital, Pauwelstrasse 30, Aachen 52074, Germany.

Received: 30 March 2012 Accepted: 21 August 2012

Published: 24 August 2012

\section{References}

1. Fukuda S, Warner DS: Cerebral protection. Br J Anaesth 2007, 99:10-17.

2. Valtysson J, Persson L, Hillered L: Extracellular ischaemia markers in repeated global ischaemia and secondary hypoxaemia monitored by microdialysis in rat brain. Acta Neurochir (Wien) 1998, 140:387-395.

3. Zoremba N, Homola A, Rossaint R, Sykova E: Brain metabolism and extracellular space diffusion parameters during and after transient global hypoxia in the rat cortex. Exp Neurol 2007, 203:34-41.

4. Sanders RD, Manning HJ, Robertson NJ, Ma D, Edwards AD, Hagberg H, Maze M: Preconditioning and postinsult therapies for perinatal hypoxic-ischemic injury at term. Anesthesiology 2010, 113:233-249.

5. Zhao Z, Corvera JS, Halkos ME, Kerendi F, Wang N, Guyton RA, VintenJohansen J: Inhibition of myocardial injury by ischemic postconditioning during reperfusion: comparison with ischemic preconditioning. Am J Physiol Heart Circ Physiol 2003, 285:H579-H588.

6. Zhao Z:Vinten-Johansen J: Postconditioning: reduction of reperfusion-induced injury. Cardiovasc Res 2006, 70:200-211.

7. Saver JL: Time is brain-quantified. Stroke 2006, 37:263-266.

8. Bernard S: Hypothermia after cardiac arrest: expanding the therapeutic scope. Crit Care Med 2009, 37:S227-\$233.

9. Hausenloy DJ, Yellon DM: The therapeutic potential of ischemic conditioning: an update. Nat Rev Cardiol 2011, 8(11):619-629.

10. Gao X, Ren C, Zhao H: Protective effects of ischemic postconditioning compared with gradual reperfusion or preconditioning. J Neurosci Res 2008, 86:2505-2511.

11. Kersten JR, Montgomery MW, Pagel PS, Warltier DC: Levosimendan, a new positive inotropic drug, decreases myocardial infarct size via activation of K(ATP) channels. Anesth Analg 2000, 90:5-11.

12. Hein M, Roehl AB, Baumert JH, Scherer K, Steendijk P, Rossaint R: Anti-ischemic effects of inotropic agents in experimental right ventricular infarction. Acta Anaesthesiol Scand 2009, 53:941-948.

13. Katircioglu SF, Seren M, Parlar Al, Turan NN, Manavbasi Y, Aydog G, Cicekcioglu F, Tutun U, Ulus AT: Levosimendan effect on spinal cord ischemia-reperfusion injury following aortic clamping. J Card Surg 2008, 23:44-48. 
14. Lafci B, Yasa H, Ilhan G, Ortac R, Yilik L, Kestelli M, Goktogan T, Gurbuz A: Protection of the spinal cord from ischemia: comparative effects of levosimendan and iloprost. Eur Surg Res 2008, 41:1-7.

15. Roehl AB, Hein M, Loetscher PD, Rossaint J, Weiss J, Rossaint R, Coburn M: Neuroprotective properties of Levosimendan in an in vitro model of traumatic brain injury. BMC Neurol 2010, 10:97.

16. Honisch A, Theuring N, Ebner B, Wagner C, Strasser RH, Weinbrenner C: Postconditioning with levosimendan reduces the infarct size involving the PI3K pathway and KATP-channel activation but is independent of PDE-III inhibition. Basic Res Cardiol 2010, 105:155-167.

17. Das B, Sarkar C: Pharmacological preconditioning by levosimendan is mediated by inducible nitric oxide synthase and mitochondrial KATP channel activation in the in vivo anesthetized rabbit heart model. Vascul Pharmacol 2007, 47:248-256.

18. Kopustinskiene DM, Pollesello P, Saris NE: Levosimendan is a mitochondrial K(ATP) channel opener. Eur J Pharmacol 2001, 428:311-314.

19. Mayanagi K, Gaspar T, Katakam PV, Busija DW: Systemic administration of diazoxide induces delayed preconditioning against transient focal cerebral ischemia in rats. Brain Res 2007, 1168:106-111.

20. Bantel C, Maze M, Trapp S: Neuronal preconditioning by inhalational anesthetics: evidence for the role of plasmalemmal adenosine triphosphate-sensitive potassium channels. Anesthesiology 2009, 110:986-995.

21. Guide for the care and use of laboratory animals: Institute of Laboratory Animal Resources, Commission on Life Sciences, National Research Council. 8th edition. Washington, DC: National Academy Press; 2011

22. Zoremba N, Homola A, Slais K, Vorisek I, Rossaint R, Lehmenkuhler A Sykova E: Extracellular diffusion parameters in the rat somatosensory cortex during recovery from transient global ischemia/hypoxia. J Cereb Blood Flow Metab 2008, 28(10):1665-1673.

23. Dethloff TJ, Knudsen GM, Larsen FS: Cerebral blood flow autoregulation in experimental liver failure. J Cereb Blood Flow Metab 2008, 28:916-926.

24. Ungerstedt U: Microdialysis-principles and applications for studies in animals and man. J Intern Med 1991, 230:365-373.

25. Blaustein MP, Lederer WJ: Sodium/calcium exchange: its physiological implications. Physiol Rev 1999, 79:763-854.

26. Kulik A, Trapp S, Ballanyi K: Ischemia but not anoxia evokes vesicular and $\mathrm{Ca}(2+)$-independent glutamate release in the dorsal vagal complex in vitro. J Neurophysiol 2000, 83:2905-2915.

27. Kunimatsu T, Asai S, Kanematsu K, Zhao H, Kohno T, Misaki T, Ishikawa K: Transient in vivo membrane depolarization and glutamate release before anoxic depolarization in rat striatum. Brain Res 1999, 831:273-282.

28. Lasarzik I, Noppens RR, Wolf T, Bauer H, Luh C, Werner C, Engelhard K, Thal SC: Dose-Dependent Influence of Sevoflurane Anesthesia on Neuronal Survival and Cognitive Outcome After Transient Forebrain Ischemia in Sprague-Dawley Rats. Neurocrit Care 2011, 15(3):577-584.

29. Iqbal S, Baziany A, Gordon S, Wright S, Hussain M, Miyashita H, Shuaib A, Hasan Rajput A: Neuroprotective effect of tiagabine in transient forebrain global ischemia: an in vivo microdialysis, behavioral, and histological study. Brain Res 2002, 946:162-170.

30. Nakayama R, Yano T, Ushijima K, Abe E, Terasaki H: Effects of dantrolene on extracellular glutamate concentration and neuronal death in the rat hippocampal CA1 region subjected to transient ischemia. Anesthesiology 2002, 96:705-710.

31. Choi SK, Lee G, Choi S, Kim YJ, Park H, Park BJ: Neuroprotective effects by nimodipine treatment in the experimental global ischemic rat model: real time estimation of glutamate. J Korean Neurosurg Soc 2011, 49:1-7.

32. Kang SW, Choi S, Park E, Chae S, Choi S, Jin Joo H, Lee G, Park H: Neuroprotective effects of magnesium-sulfate on ischemic injury mediated by modulating the release of glutamate and reduced of hyperreperfusion. Brain Res 2011, 1371:121-128.

33. Domoki F, Perciaccante JV, Veltkamp R, Bari F, Busija DW: Mitochondrial potassium channel opener diazoxide preserves neuronal-vascular function after cerebral ischemia in newborn pigs. Stroke 1999 30:2713-8-2718-9. discussion.

34. Robin E, Simerabet M, Hassoun SM, Adamczyk S, Tavernier B, Vallet B, Bordet R, Lebuffe G: Postconditioning in focal cerebral ischemia: role of the mitochondrial ATP-dependent potassium channel. Brain Res 2011 1375:137-146.
35. Yamada K, Ji JJ, Yuan H, Miki T, Sato S, Horimoto N, Shimizu T, Seino S, Inagaki N: Protective role of ATP-sensitive potassium channels in hypoxia-induced generalized seizure. Science 2001, 292:1543-1546.

36. Grover GJ, Newburger J, Sleph PG, Dzwonczyk S, Taylor SC, Ahmed SZ, Atwal KS: Cardioprotective effects of the potassium channel opener cromakalim: stereoselectivity and effects on myocardial adenine nucleotides. J Pharmacol Exp Ther 1991, 257:156-162.

37. Antila $S$, Huuskonen $H$, Nevalainen $T$, Kanerva $H$, Vanninen $P$, Lehtonen $L$ : Site dependent bioavailability and metabolism of levosimendan in dogs. Eur J Pharm Sci 1999, 9:85-91.

38. Abo-Ramadan U, Durukan A, Pitkonen M, Marinkovic I, Tatlisumak E, Pedrono E, Soinne L, Strbian D, Tatlisumak T: Post-ischemic leakiness of the blood-brain barrier: a quantitative and systematic assessment by Patlak plots. Exp Neurol 2009, 219:328-333.

39. Koizumi H, Fujisawa H, Suehiro E, Shirao S, Suzuki M: Neuroprotective effects of ebselen following forebrain ischemia: involvement of glutamate and nitric oxide. Neurol Med Chir (Tokyo) 2011, 51:337-343.

40. Munakata H, Okada K, Hasegawa T, Hino Y, Kano H, Matsumori M, Okita Y: Controlled low-flow reperfusion after warm brain ischemia reduces reperfusion injury in canine model. Perfusion 2010, 25:159-168.

41. Bravo MC, Lopez P, Cabanas F, Perez-Rodriguez J, Perez-Fernandez E, Quero J, Pellicer A: Acute effects of levosimendan on cerebral and systemic perfusion and oxygenation in newborns: an observational study. Neonatology 2011, 99:217-223.

42. Cengiz SL, Erdi MF, Tosun M, Atalik E, Avunduk MC, Sonmez FC, Mehmetoglu I, Baysefer A: Beneficial effects of levosimendan on cerebral vasospasm induced by subarachnoid haemorrhage: an experimental study. Brain Inj 2010, 24:877-885.

43. Lenzser G, Kis B, Bari F, Busija DW: Diazoxide preconditioning attenuates global cerebral ischemia-induced blood-brain barrier permeability. Brain Res 2005, 1051:72-80.

44. Zhu H, Luo W, Wang H: Iptakalim protects against hypoxic brain injury through multiple pathways associated with ATP-sensitive potassium channels. Neuroscience 2008, 157:884-894

45. Denes A, Ferenczi S, Kovacs KJ: Systemic inflammatory challenges compromise survival after experimental stroke via augmenting brain inflammation, blood- brain barrier damage and brain oedema independently of infarct size. J Neuroinflammation 2011, 8:164.

doi:10.1186/1471-2377-12-81

Cite this article as: Roehl et al:: The effects of levosimendan on brain metabolism during initial recovery from global transient ischaemia/ hypoxia. BMC Neurology 2012 12:81.

\section{Submit your next manuscript to BioMed Central and take full advantage of:}

- Convenient online submission

- Thorough peer review

- No space constraints or color figure charges

- Immediate publication on acceptance

- Inclusion in PubMed, CAS, Scopus and Google Scholar

- Research which is freely available for redistribution 